\title{
Diagnosis and management of $a_{1}$-antitrypsin deficiency in Europe: an expert survey
}

\author{
Ildikó Horváth, Maria Canotilho², Jan Chlumský3, Joanna Chorostowska- \\ Wynimko ${ }^{4}$, Luciano Corda ${ }^{5}$, Eric Derom ${ }^{6}$, Joachim H. Ficker ${ }^{7}$, \\ Meinhard Kneussl ${ }^{8}$, Marc Miravitlles $\mathbb{1}^{9}$, Maria Sucena ${ }^{10}$, Gabriel Thabut ${ }^{11}$, \\ Alice M. Turner (1) ${ }^{12}$, Emily van 't Wout ${ }^{13}$ and N. Gerard McElvaney ${ }^{14}$
}

Affiliations: ${ }^{1}$ Dept of Pulmonology, National Koranyi Institute for Pulmonology in Budapest, Budapest, Hungary. ${ }^{2}$ Dept of Pneumology, Hospital of Santo Andre - Centro Hospitalar de Leiria, Leiria, Portugal. ${ }^{3}$ Dept of Pneumology, Thomayer Hospital, First Faculty of Medicine, Charles University, Prague, Czech Republic. ${ }^{4}$ Dept of Genetics and Clinical Immunology, National Institute of Tuberculosis and Lung Diseases, Warsaw, Poland. ${ }^{5}$ Dept of Internal Medicine, Respiratory Disease Unit, Spedali Civili, Brescia, Italy. ${ }^{6}$ Dept of Respiratory Medicine, Ghent University Hospital, Ghent, Belgium. ${ }^{7} 3 \mathrm{rd}$ Medical Dept, Nuremberg General Hospital/ Paracelsus Medical University, Nuremberg, Germany. ${ }^{8}$ Dept of Internal Medicine II and Pneumology at Wilhelminenspital Wien, Vienna, Austria. 'Pulmonology Dept, University Hospital Vall d'Hebron, CIBER de Enfermedades Respiratorias (CIBERES), Barcelona, Spain. ${ }^{10}$ Pulmonology Dept, Centro Hospitalar de São João, Porto, Portugal. ${ }^{11}$ Dept of Pneumology and Lung Transplantation, Bichat Hospital, Paris, France. ${ }^{12}$ Institute of Applied Health Research, University of Birmingham, Birmingham, UK. ${ }^{13}$ Leiden University Medical Centre, Dept of Pulmonology, Leiden, Netherlands. ${ }^{14}$ Dept of Respiratory Medicine, Beaumont Hospital, Royal College of Surgeons in Ireland, Dublin, Ireland.

Correspondence: Ildikó Horváth, Department of Pulmonology of the National Koranyi Institute for Pulmonology in Budapest, Budapest, Hungary. E-mail: ildiko.horvathakoranyi.hu.

ABSTRACT Despite recent improvements, $\alpha_{1}$-antitrypsin deficiency (AATD) remains a rarely diagnosed and treated condition. To assess the variability of AATD diagnosis/treatment in Europe, and to evaluate clinicians' views on methods to optimise management, specialist AATD clinicians were invited to complete a web-based survey.

Surveys were completed by 15 physicians from 14 centres in 13 European countries. All respondents perceived the AATD diagnosis rate to be low in their country; $77 \%$ of physicians believed that $\sim 15 \%$ of cases were diagnosed. Low awareness was perceived as the greatest barrier to diagnosis. Spirometry was considered more practical than quantitative computed tomography (QCT) for monitoring AATD patients in clinical practice; QCT was considered more useful in trials. AAT therapy provision was reported to be highly variable: France and Germany were reported to treat the highest proportion $(\sim 60 \%)$ of diagnosed patients, in contrast to the UK and Hungary, where virtually no patients receive AAT therapy. Most clinicians supported self-administration and extended dosing intervals to improve convenience of AAT therapy.

This survey indicates that AATD diagnosis and management are highly heterogeneous in Europe; European cooperation is essential to generate data to support access to AAT therapy. Improving convenience of AAT therapy is an ongoing objective.

@ERSpublications

Access to $\alpha_{1}$-antitrypsin $\left(\alpha_{1}-\mathrm{AT}\right)$ therapy varies in Europe; where available, $\alpha_{1}$-AT therapy optimisation is the goal http://ow.ly/YL6m30n4LV3

Cite this article as: Horváth I, Canotilho M, Chlumský J, et al. Diagnosis and management of $\alpha_{1}$-antitrypsin deficiency in Europe: an expert survey. ERJ Open Res 2019; 5: 00171-2018 [https:// doi.org/10.1183/23120541.00171-2018]. 


\section{Introduction}

$\alpha_{1}$-Antitrypsin deficiency (AATD) is a well-established, but underdiagnosed inherited condition that can lead to emphysema and liver disease. It is caused by mutations in the SERPINA1 gene encoding $\alpha_{1}$-antitrypsin (AAT), a key serum protease inhibitor. In individuals with AATD, serine proteases, primarily neutrophil elastase, are not inhibited, resulting in degradation of lung tissue and eventual progression to emphysema [1]. In addition, patients with the $\mathrm{Z}$ variant or rare variants such as $\mathrm{M}_{\mathrm{Malton}}$ or $\mathrm{S}_{\text {iiyam }}$ have an increased risk of developing liver disease, owing to protein accumulation in hepatocytes [1].

Multiple factors contribute to the underdiagnosis of AATD, including similarities in presentation to general chronic obstructive pulmonary disease (COPD) and asthma and lack of access to testing, with low disease awareness perhaps the key issue [2-5]. Targeted detection programmes aimed at symptomatic individuals, e.g. COPD patients, and neonatal screening have been employed in Europe and have helped to increase diagnosis rates [6-8]. However, active screening programmes for AATD do not exist in many countries and numerous patients in Europe remain undiagnosed. Due to the progressive and irreversible destruction of lung architecture in AATD, early detection is essential to enable lifestyle modifications (e.g. smoking cessation) and appropriate treatment [9].

COPD related to AATD is managed symptomatically with bronchodilators [10], in line with non-AATD-associated COPD. However, purified human AAT is the only disease-modifying therapy currently available that can slow progression of emphysema related to AATD [11, 12]. Currently, the therapy is recommended only for patients with severe deficiency genotypes, e.g. $\mathrm{PI}^{\star} \mathrm{ZZ}$ (European Respiratory Society (ERS) guidelines and US guidelines) and PI*SZ (US guidelines only) [13, 14]. The optimal time for treatment initiation has been greatly debated. Recent US guidelines suggest that i.v. AAT therapy can be considered in symptomatic individuals at any level of spirometric impairment, as determined by forced expiratory volume in $1 \mathrm{~s}$ (FEV1) \% predicted, although the strongest recommendation for treatment is when FEV1 is $\leqslant 65 \%$ pred [13]. The recent ERS statement does not specify a threshold for treatment [14]. The 2003 statement from the American Thoracic Society (ATS) and ERS recommended AAT therapy in patients with moderate airflow obstruction (e.g. FEV1 35-60\% pred), because historically there is more evidence of an effect on spirometric decline in this range [1].

Current trends are moving towards a personalised approach to AAT therapy provision [15], with pharmacokinetic models demonstrating that extended dosing intervals are feasible [16]. Despite this, there are few recommendations on methods to improve convenience of i.v. dosing regimens, e.g. extended-interval dosing and self-administration. However, in many European countries, accessing AAT therapy is the principal challenge - half the European countries surveyed in the latest ERS statement reported having no access or very limited access to treatment [14].

To gain an understanding of the current status of AATD diagnosis and management in Europe, as well as attitudes towards methods for AAT therapy optimisation, we performed a survey of European AATD experts.

\section{Materials and methods}

Data collection

Clinicians treating AATD from across Europe were invited to complete a web-based survey. The objective was to gather expert opinion on the diagnosis and management of patients with AATD. The survey consisted of 58 questions covering 1) size of patient population; 2) diagnosis and management of AATD; 3) AATD treatment options; 4) dosing of AAT therapy; and 5) self-administration and home treatment with AAT therapy. Self-administration was defined as i.v. administration of AAT performed by the patient or a non-professional (e.g. assistance from a spouse or relative). Home treatment was defined as i.v. administration of AAT performed by a healthcare professional (e.g. physician or nurse).

Data analysis

Descriptive statistics only are reported; no formal statistical tests were performed.

\section{Results}

\section{Survey representation}

Completed surveys were returned by 15 physicians from 14 centres in 13 countries: Austria, Belgium, Czech Republic, France, Germany, Hungary, Ireland, Italy, the Netherlands, Poland, Portugal, Spain and the UK. All respondents are responsible for managing and treating patients with severe AATD genotypes, e.g. $\mathrm{PI}^{\star} \mathrm{ZZ}$.

\section{Diagnosis of AATD}

Physicians' estimates of the number of diagnosed AATD cases varied greatly between countries and were generally far lower than the number of individuals with severe AATD in each country based on published estimates (table 1) $[17,18]$. All respondents perceived the rate of diagnosis to be low in their countries: 
TABLE 1 Testing for $\mathrm{a}_{1}$-antitrypsin deficiency (AATD) and the estimated number of cases in Europe

\begin{tabular}{|c|c|c|c|c|c|}
\hline & \multicolumn{2}{|c|}{ National AATD treatment infrastructure } & \multicolumn{3}{|c|}{$\begin{array}{l}\text { Estimated number of diagnosed AATD cases } \\
\text { versus overall number of severe deficiency carriers }\end{array}$} \\
\hline & $\begin{array}{l}\text { Nationwide active } \\
\text { screening programme }\end{array}$ & $\begin{array}{l}\text { Treatment centres } \\
\text { with }>10 \text { patients } n\end{array}$ & $\begin{array}{l}\text { Estimated diagnosed } \\
\text { severe AATD cases } n\end{array}$ & \multicolumn{2}{|c|}{ Individuals with severe AATD genotype ${ }^{\#}$} \\
\hline Czech Republic & No & 1 & 70 & N/D & N/D \\
\hline France & No & $\geqslant 5$ & $\geqslant 1000$ & 9982 (7662-12989) & $17191(13255-22270)$ \\
\hline Germany & No & $\geqslant 5$ & $\geqslant 1000$ & $8003(5520-11577)$ & $20611(13380-31626)$ \\
\hline Hungary & Yes & 0 & $\leqslant 50$ & 458 (149-1352) & N/D \\
\hline Ireland & Yes & 0 & 300 & N/D & 2265 (1264-4019) \\
\hline Italy & No & 1 & 500 & $15659(2864-19150)$ & $10652(7046-16049)$ \\
\hline The Netherlands & No & 1 & 500 & $1711(976-2980)$ & 5353 (3057-9298) \\
\hline & & & & & $\begin{array}{l}\text { Scotland: } 344 \\
\text { (182-644) }\end{array}$ \\
\hline
\end{tabular}

$77 \%$ of physicians believed that $\sim 15 \%$ of cases had been diagnosed, with the remainder estimating a $30 \%$ diagnosis rate. Few AATD screening programmes were reported in Europe (table 1); all respondents had access to AAT serum level testing and phenotyping (isoelectric focusing), 93\% had access to targeted genotyping for common deficiency variants and $73 \%$ had access to SERPINA1 gene sequencing (to identify rare/novel/null variants). The majority of physicians (60\%) estimated that on average it took $\geqslant 5$ years to obtain a diagnosis of AATD from first onset of symptoms (figure 1a); lack of awareness was most frequently cited as the biggest barrier to AATD diagnosis (77\% of physicians) (figure $1 \mathrm{~b}$ ).

\section{Treatment and monitoring of AATD}

Across Europe, patients with AATD were reported to be symptomatically managed principally with long-acting $\beta$-adrenergic receptor agonists, long-acting muscarinic receptor agonists and inhaled corticosteroids. Provision of AAT therapy is highly variable throughout Europe. France and Germany were reported to have the highest proportions of diagnosed AATD patients receiving AAT therapy (60\%); in Spain, $\sim 20 \%$ of patients receive treatment with AAT (figure 2).

a)

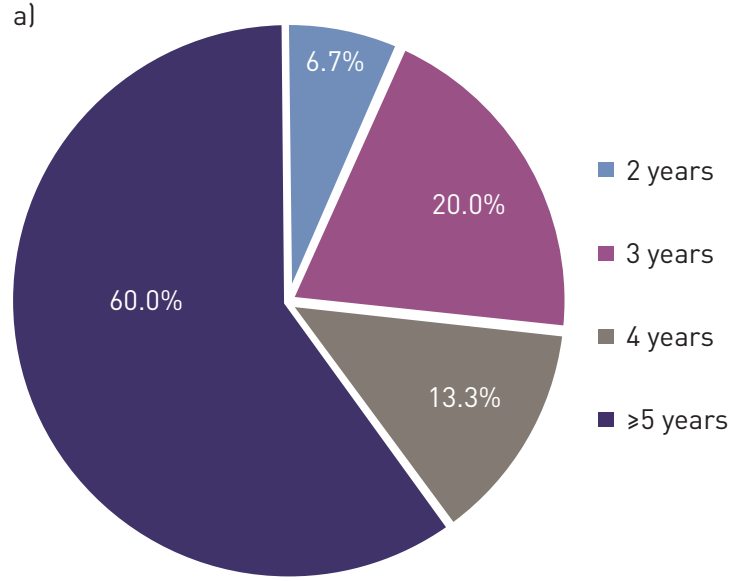

b)

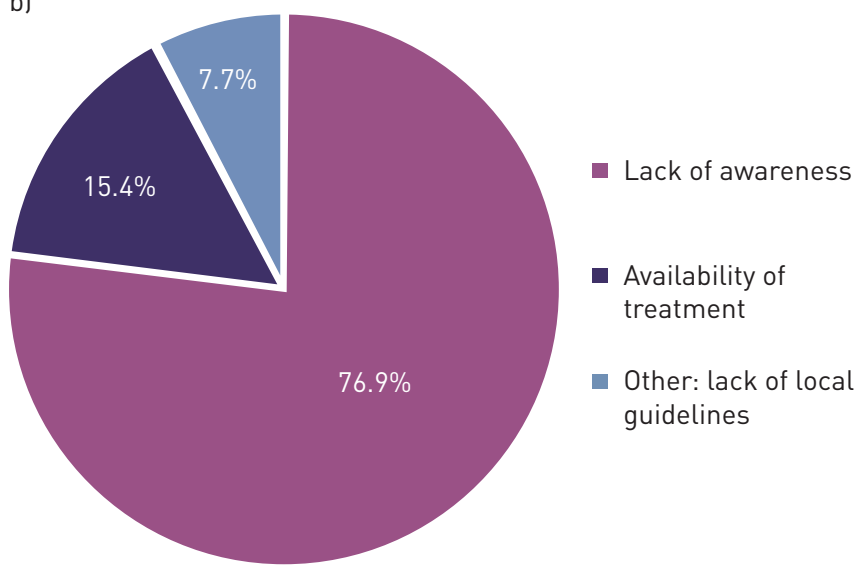

FIGURE 1 a) Estimated number of years to obtain formal diagnosis of $a_{1}$-antitrypsin deficiency (AATD), from first onset of symptoms; b) barriers to diagnosis of AATD in Europe. 
AAT therapy not reimbursed

AAT therapy partially reimbursed or with conditions

AAT therapy reimbursed

No information available

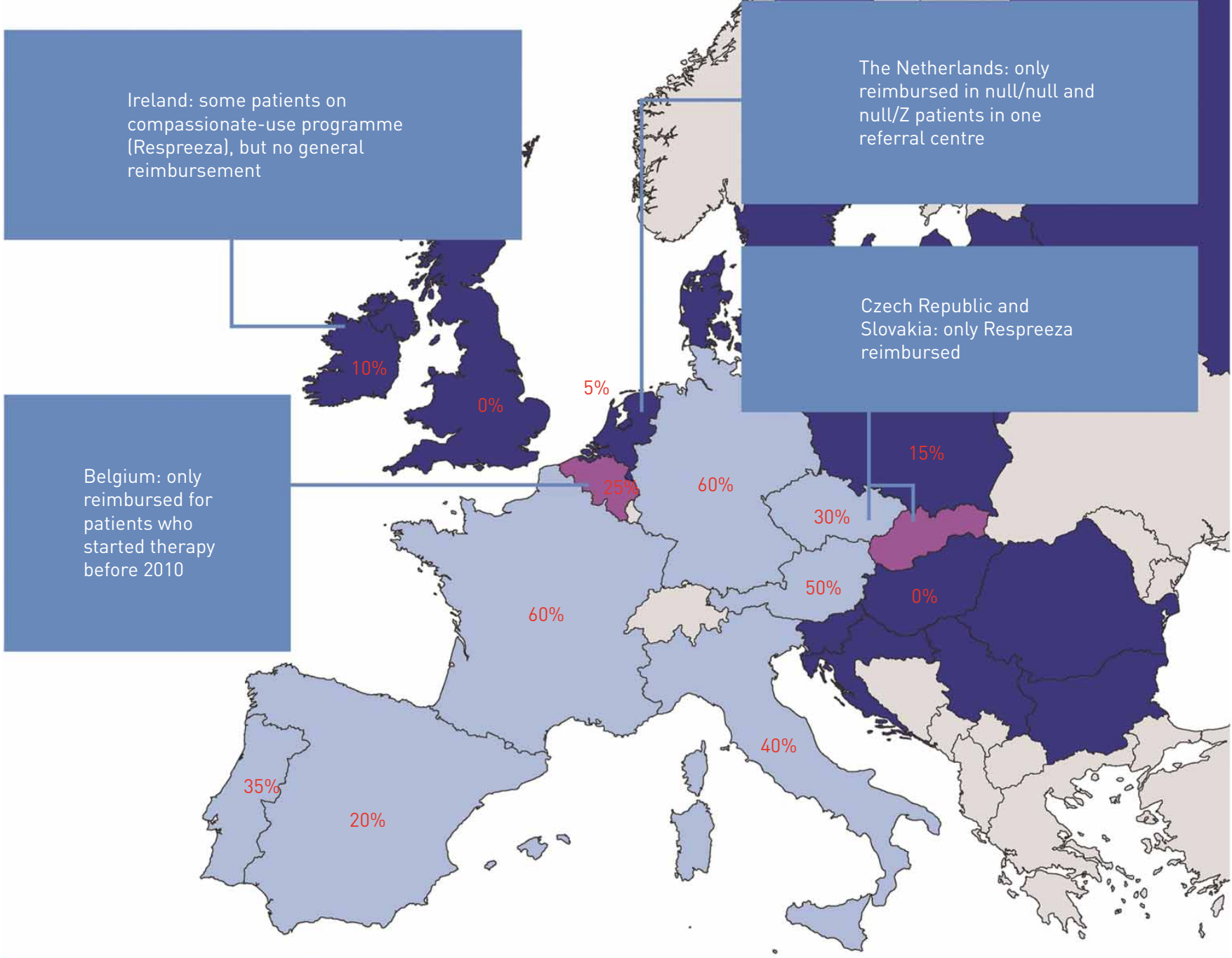

\begin{tabular}{|c|c|}
\hline Product & Countries with regulatory approval \\
\hline Alfalastin & France \\
\hline Prolastin & $\begin{array}{l}\text { Austria, Belgium, Czech Republic, Denmark, Finland, France, Germany, Greece, Ireland, Italy, } \\
\text { the Netherlands, Norway, Poland, Portugal, Slovakia, Spain, Sweden and Switzerland }\end{array}$ \\
\hline Respreeza & EU-wide \\
\hline
\end{tabular}

FIGURE $2 a_{1}$-Antitrypsin (AAT) therapy availability across Europe. Reimbursement data obtained from European Respiratory Society 2017 statement [14]. Data are presented as percentage of diagnosed patients on AAT therapy; values based on individual physician estimates. Portugal figure represents the average of two responses. 


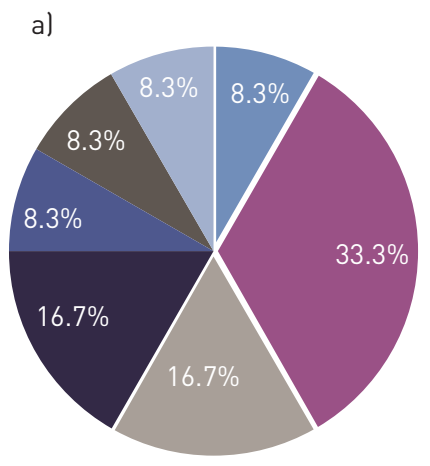

- Large infusion volume

- Lengthy infusion time

- Available vial size

- Other: frequency of infusions

- Other: outpatient clinic overbooked, difficult to schedule

- Other: treatment provided by one centre only

- Other: the need for i.v. infusions

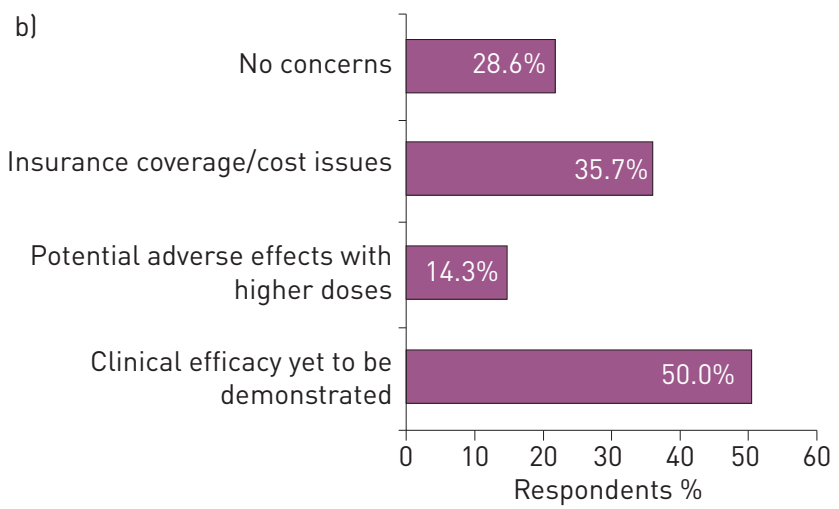

FIGURE 3 a $\mathrm{a}_{1}$-Antitrypsin (AAT) therapy considerations: a) practical difficulties with AAT infusions; b) concerns regarding AAT doses $>60$ mg $\cdot \mathrm{kg}^{-1}$ per week. Data are presented as percentage of respondents; physicians could choose more than one concern regarding AAT doses $>60 \mathrm{mg} \cdot \mathrm{kg}^{-1}$. No AAT products were specified and there was no agreed definition on what constituted a lengthy infusion time.

\section{AAT therapy considerations}

Practical challenges with AAT infusions were reported, the most often cited being infusion time (33\%; figure 3a). The majority of respondents (73\%) would consider alternative dosing strategies (e.g. bi-weekly dosing); reasons included to cover holidays and for individuals in full-time employment. This practice is already employed in some countries, e.g. all patients in the Czech Republic receive bi-weekly dosing, and it is an option in France and Spain. Most respondents (87\%) would consider providing AAT doses higher than the recommended weekly dose of $60 \mathrm{mg} \cdot \mathrm{kg}^{-1}$ in some situations, such as when the "protective threshold" for AAT serum level was not reached, during exacerbations and for patients with rapidly deteriorating disease. The main concern regarding higher doses was the lack of proven clinical efficacy; however, nearly one-third of respondents (29\%) had no concerns (figure $3 \mathrm{~b}$ ).

\section{Monitoring of AATD}

Physicians were asked what they perceived to be the most useful methods for monitoring disease progression and treatment efficacy in diagnosed AATD patients (figure 4). Quantitative computed tomography (QCT) of the lungs, i.e. measurement of lung density, was viewed as the most useful measure in clinical trial settings. Diffusing capacity of the lung for carbon monoxide (DLCO) was considered the most useful measure for monitoring AATD in routine clinical practice, but less useful in clinical trials. Some physicians commented that DLCO can be very informative in trials involving fewer or single centres. FEV1 was considered less useful than QCT and DLCO in trials, but more practical than QCT in routine clinical practice.

\section{Optimising treatment of AATD}

Timing of treatment initiation

Most physicians surveyed would consider using AAT therapy in patients with moderate disease severity, i.e. $\mathrm{FEV} 1<80 \%$ and $\geqslant 35 \%$ pred (figure 5). A minority would consider AAT in early- and late-/end-stage disease ( $\mathrm{FEV}_{1} \geqslant 80 \%$ and $<35 \%$ pred). Some physicians commented that in patients with severe AATD and

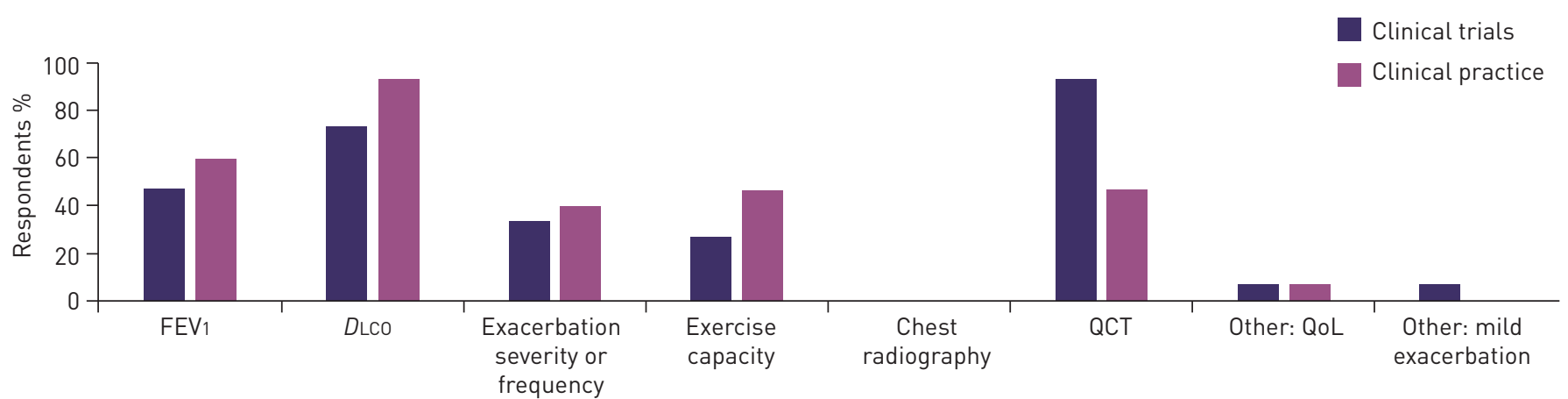

FIGURE 4 Physician perspectives on the most useful methods for monitoring $a_{1}$-antitrypsin deficiency in the clinical trial setting versus clinical practice. FEV1: forced expiratory volume in $1 \mathrm{~s}$; DLCO: diffusing capacity of the lung for carbon monoxide; QCT: quantitative computed tomography; QoL: quality of life. 
FIGURE 5 Degree of lung function impairment at which physicians would consider commencing $a_{1}$ antitrypsin therapy. FEV1: forced expiratory volume in $1 \mathrm{~s}$.

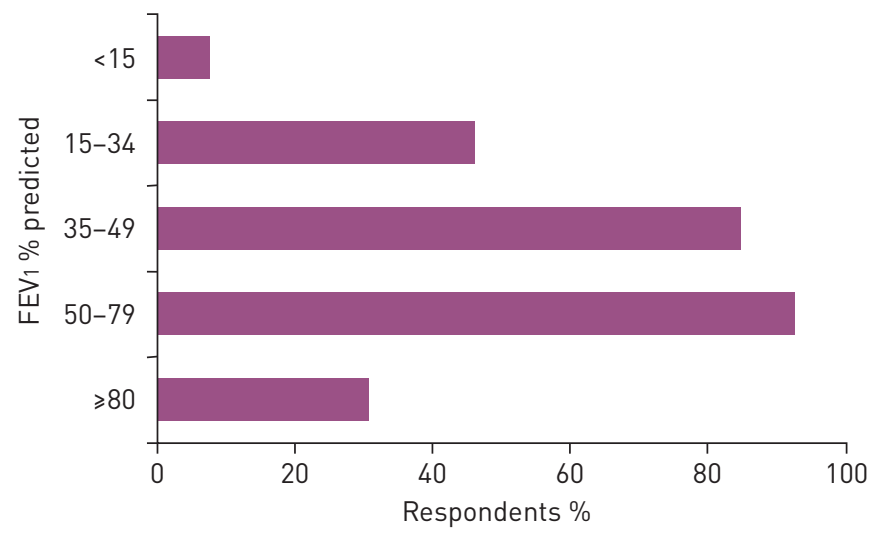

FEV 1 above the historically recommended range for treatment (FEV1 35-60\% pred) [1], QCT should be used to confirm the presence, severity and distribution of emphysema.

\section{Self-administration}

Self-administration of i.v. AAT was not available in any of the countries surveyed. However, all respondents would consider self-administration for some patients if it were available. Respondents suggested that a number of patient groups would benefit from self-administration, e.g. those in employment (figure 6a). Overall, $\sim 50 \%$ of respondents felt that for patients to self-administer independently, three training sessions would be required (figure 6b); $86 \%$ of respondents felt that training should be provided by hospital-based respiratory nurses. Greater independence was viewed as the most important advantage of self-administration; safety issues surrounding i.v. administration were viewed as the main disadvantage.

\section{Home treatment}

Home treatment was available in four of the countries surveyed (Ireland, France, Poland, Czech Republic), provided by trained community nurses, but only as part of clinical trials in Ireland and Poland. Home therapy is the only treatment option in Ireland and France; in Poland, regular treatment is provided at home, but is also available at hospital outpatient clinics. Convenience for the patient was viewed as the most important advantage of home treatment; fewer resources available in the event of an emergency was most frequently cited as the main disadvantage.

\section{Discussion}

This survey of experienced clinicians treating AATD indicates that levels of diagnosis and disease awareness in Europe remain low. Furthermore, there is great variability across Europe in the provision of AAT therapy, the only pharmacological intervention able to slow emphysema progression related to AATD.

a)

$“<50$ years old, full-time job, compliant"

“A disabled, older patient living a distance from the hospital or a young patient still in work"

"Young, working person with poor access to the hospital"

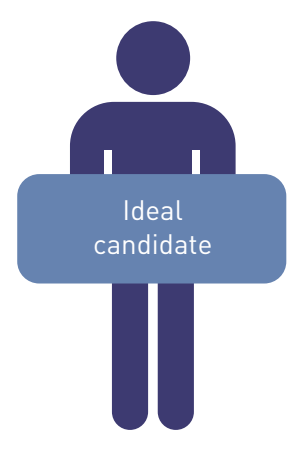

b)

"Patients without cognitive impairment in work who have the ability to work cleanly and efficiently"

“An older, active patient, potentially receiving oxygen therapy"

“Young, active, mobile, in employment, with family"

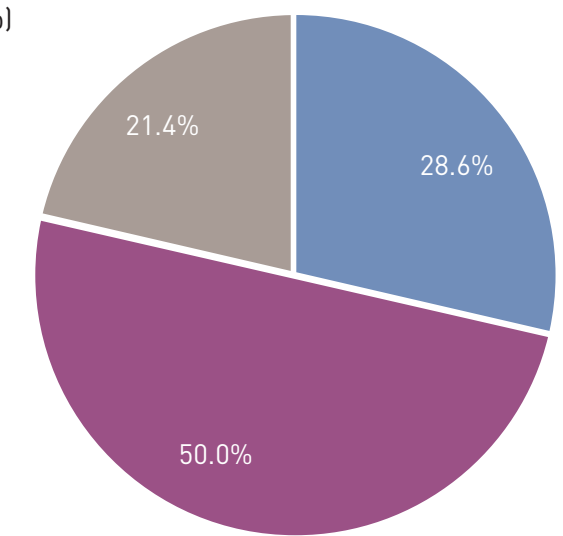

$2 \square 3 \square \geqslant 5$

FIGURE 6 Physician perspectives on self-administration of i.v. $a_{1}$-antitrypsin. a) The ideal candidate for self-administration; b) the number of training sessions required for a patient to self-administer independently. Data are presented as percentage of respondents. 
Published reports $[17,18]$ provide high estimates of the number of $\mathrm{PI}^{\star} \mathrm{ZZ}$ carriers in Europe; although these do not reflect the number of symptomatic cases, the estimated numbers of diagnosed cases reported in our survey are lower than might be expected. This disparity may be due to the number of patients who are currently asymptomatic [19]; however, it is likely that many patients are misdiagnosed or face delays in diagnosis $[2,4]$. Most physicians surveyed believed that only $15 \%$ of AATD cases had been identified, and that a confirmed diagnosis of AATD takes $>5$ years to obtain. A similar scenario was reported in Canada, with a delay in diagnosis of 7 years [20]. Our survey suggests that low awareness remains the biggest contributor to underdiagnosis/delay in diagnosis in Europe, consistent with previous studies [4, 5, 21]. In Europe, an overarching issue is that awareness and detection in primary care are very low, with few physicians referring patients for AATD testing [4, 21]. Nevertheless, there have been successes with public health campaigns focused on improving diagnosis. For example, in Germany, a disease-awareness campaign coupled with AATD testing dramatically improved AATD detection [6].

Although AATD screening programmes in Europe have helped to increase detection of AATD and improve access to AAT therapy [7, 22-25], only four national screening programmes were reported in our survey (table 1). There are several screening strategies for AATD, including population-based screening, targeted screening of COPD patients, testing patients with liver disease and familial screening. Population-based screening is uncommon and one of the few implementations of this approach was the neonatal screening programme employed by Sweden in the 1970s. This programme provided important insights into the natural history of AATD and the variability of presentation; in addition, awareness of the condition enabled patients to avoid respiratory risk factors, e.g. smoking [7]. However, population-based screening programmes can be prohibitively expensive and therefore their use has been limited to high-risk areas, e.g. in remote/isolated communities [26]. Currently, targeted screening programmes are viewed as the most feasible and economically viable approach [24], with these enriched populations leading to far higher AATD detection rates [8]. Guidelines recommend that several respiratory conditions (e.g. emphysema, bronchiectasis) and nonrespiratory conditions (e.g. liver disease, panniculitis) should trigger testing, and the strongest universal recommendation is to test all newly diagnosed COPD patients [1, 13, 27, 28]. Furthermore, owing to Mendelian inheritance, familial screening provides the highest likelihood of uncovering additional AATD cases. All guidelines advocate familial testing, with testing of siblings being the highest priority $[1,13,14]$. Testing recommendations from guidelines/statements are outlined in table 2 .

Regarding monitoring of disease progression in AATD, the recent ERS statement suggests that patients be assessed regularly by a multidisciplinary team in the first year after diagnosis [14]. Our survey revealed lung function testing via DLCO and FEV1 to be central to AATD monitoring in European clinics. This is in line with available guidance (table 2) $[1,13]$. A recommendation in guidelines from the US Alpha-1 Foundation is to conduct a baseline computed tomography (CT) scan as part of the initial assessment of AATD patients [13]. The guidelines do not recommend serial CT scanning owing to the associated radiation dosage and a lack of clarity on how best to use scan data for clinical management. Survey respondents generally felt that QCT was more suited to studies in AATD, and less applicable in clinical practice. Although lung densitometry by CT has been validated against lung pathology/function and health status, and has been the main outcome measure in clinical trials of AAT therapy, there are practical issues that reduce its applicability to routine practice [29]. Many centres do not have the requisite equipment/software to routinely conduct lung density assessment [9]. However, lung density data from clinical practice, such as that collated by the UK Antitrypsin Deficiency Assessment and Programme for Treatment (ADAPT) registry [30], have been extremely useful for research purposes, and could help to enhance the evidence base for AAT therapy. Regarding other approaches, some respondents indicated that monitoring quality of life was beneficial. Data from a recent meta-analysis indicate a possibility that AAT therapy has some efficacy on St George's Respiratory Questionnaire (SGRQ), a commonly used quality-of-life measure in respiratory disease [31]. However, this finding was associated with considerable uncertainty and further studies are required.

One aim of our survey was to assess how and when physicians utilise AAT therapy. Currently, AAT therapy is recommended only for symptomatic AATD patients, e.g. patients with airflow obstruction and physiological signs of emphysema $[1,13]$. The ATS/ERS statement from 2003 recommended that therapy be utilised between a defined range of $\mathrm{FEV} 1$ deterioration (35-60\% pred); however, today there is less emphasis placed on FEV1 ranges, and the recent ERS statement does not refer to this as a criterion for treatment [14]. Nonetheless, insurance coverage may still be linked to FEV1 deterioration, and most physicians surveyed would not consider treatment at all levels of FEV1 decline. However, some respondents would consider providing AAT in early-stage disease. Recent evidence provides a strong rationale for early intervention in AATD: data from the RAPID clinical trial programme suggest improved outcomes with early AAT therapy, with a discernible effect on lung density decline observed regardless of baseline FEV1 [12, 32, 33]. 
TABLE 2 Comparison of recommendations from American Thoracic Society (ATS)/European Respiratory Society (ERS) guidelines (2003), US Alpha-1 Foundation guidelines (2016) and ERS statement (2017)

\section{ATS/ERS statement (2003) [1]}

US Alpha-1 Foundation guidelines (2016) [13]

\section{ERS statement (2017) [14]}

Establishing AAT serum levels is a crucial first test, but must be supported by qualitative evidence of a mutation

Phenotyping and genotyping can both be used to establish mutation(s) present

Gene sequencing is necessary when a null or rare variant is suspected

\section{Who should be}

tested?

Testing for index Emphysema with early onset ( $<45$ years) case or without recognised risk factors, e.g. smoking, or with basilar hyperlucency

Bronchiectasis or liver disease with unknown aetiology

Necrotising panniculitis and anti-proteinase 3-positive vasculitis

Family history of emphysema, bronchiectasis, panniculitis or liver disease

Familial testing Recommended for siblings of index cases with severe deficiency

Should be discussed with offspring or distant relatives of individuals with severe deficiency, and siblings, offspring, parents and distant relatives of individuals with intermediate deficiency

Population screening

Screening of any age group should be discouraged; screening of active smokers with normal spirometry is not recommended

Monitoring

Full lung function testing at baseline and spirometry at yearly intervals

Regular liver function testing requires investigation

Baseline and annual spirometry

CT scan at baseline; serial CT scanning not recommended

Monitor for liver disease annually: liver ultrasound and AST, ALT, GGT, albumin, bilirubin and INR

\section{regardless of age/ethnicity}

All individuals with liver disease of unknown aetiology

All patients with granulomatosis with polyangiitis/necrotizing panniculitis

Parents, sibling, offspring and extended family should be offered genetic counselling and testing

AAT level testing alone is not recommended when testing family members

\section{AAT therapy}

utilisation

Recommended

Not

recommended
Symptomatic individuals with FEV 1 $35-65 \%$ pred

$\mathrm{PI} * \mathrm{MZ}$ individuals and current smokers
Symptomatic individuals with $\mathrm{FEV} 1$ $\leqslant 65 \%$ pred (strongest recommendation); treatment can be considered outside of this range

PI*MZ and current smokers

Emphysema/ bronchiectasis without airflow obstruction
Test parents of index case only if a null gene is suspected

Test partner of index case; if $\mathrm{PI} M \mathrm{MZ}$ is found, test offspring of index case

Test sibling(s) of index case; if $\mathrm{PI}$ MZ is found, test partner of sibling(s), if further $\mathrm{PI}{ }^{*} \mathrm{MZ}$ is found, test offspring of sibling(s)

Importance of multidisciplinary approach highlighted

Baseline: assess lung physiology and conduct routine liver function and blood tests

Up to 3 months: reassess and collate data; monitor exacerbation diary, initiate smoking cessation

Up to 6 months: assess full physiology, QoL assessment, routine blood tests

6-12 months: continue monitoring, initiate AAT therapy as appropriate

FEV 1 and DLCO are useful to monitor disease progression

Symptomatic individuals (no FEV1 range given!

$\mathrm{PI}{ }^{*} \mathrm{MZ}, \mathrm{PI}{ }^{*} \mathrm{SZ}$ and current smokers

AAT: $a_{1}$-antitrypsin; COPD: chronic obstructive pulmonary disease; CT: computed tomography; AST: aspartate aminotransferase; ALT: alanine aminotransferase; GGT: y-glutamyltransferase; INR: international normalised ratio; QoL: quality of life; FEV1: forced expiratory volume in $1 \mathrm{~s}$; $D\llcorner c o$ : diffusing capacity of the lung for carbon monoxide 
The standard dose for AAT therapy is $60 \mathrm{mg} \cdot \mathrm{kg}^{-1}$ per week; higher AAT doses are not currently licensed or recommended. However, this survey indicates that some physicians would consider providing higher doses, e.g. at times of exacerbation or for patients with fast-declining lung function. There is increasing interest in higher AAT doses: a recent pilot study found that normalising serum AAT levels in AATD patients by increasing dosing from 60 to $120 \mathrm{mg} \cdot \mathrm{kg}^{-1}$ per week further reduced markers of residual proteolytic and inflammatory activity [34]. In addition, an analysis of patients who received $120 \mathrm{mg} \cdot \mathrm{kg}^{-1}$ AAT or placebo to cover 2-week periods in the RAPID clinical trial programme found no increase in the infusion-adjusted event rate at $24 \mathrm{~h}$ and $72 \mathrm{~h}$ after administration [35]. The clinical utility and long-term safety of higher doses require further study and trials are ongoing.

This survey highlights that access to AAT therapy is not uniform throughout Europe, with some countries reporting that no diagnosed AATD patients receive AAT therapy. Indeed, the recent ERS statement suggests that reimbursement is an ongoing challenge in Europe (figure 2) [14]. The European situation reflects the Canadian experience, where large disparities in the provision of AAT therapy were observed between provinces [20]. Within the European Union (EU), policies exist to support equal access to novel therapies between countries. For example, directive 89/105/EEC6 states that pricing and reimbursement decisions must be made in a timely and transparent manner [36]. Nevertheless, these decisions are ultimately at the discretion of member states according to their independent appraisal of the technology and budgetary requirements [37]. In addition, although EU polices support cross-border diagnosis and treatment in rare diseases (EU directive 2011/24/EU), the health authority or insurance provider in the home country must agree to reimburse treatment in the destination country [37]. This issue, coupled with the impracticality of travelling abroad for medical treatment, suggests that cross-border treatment is not a long-term solution to address variation in access to AAT therapy in Europe.

Continuing to build the evidence base for AAT therapy is essential to support access to treatment. The RAPID clinical trial programme demonstrated that AAT therapy is effective and disease-modifying in AATD $[11,12,33]$. Beyond this, some authorities desire data on patient-centred outcomes, e.g. mortality and QoL, to support the pharmacoeconomic profile of AAT therapy [38]. However, it is difficult to generate these data in clinical trials, as large numbers of AATD patients would have to be followed for long durations. Patient registries in Europe are therefore crucial in gathering longitudinal data of the efficacy of AAT therapy on these outcomes [14].

Where AAT therapy is available, improving convenience is a key challenge. Alternative dosing strategies (e.g. bi-weekly) and home treatment are already used in some European countries [28, 39]. In addition to the national home treatment programmes reported here, a third party-sponsored Berlin-based programme in Germany has reported successful results in terms of improving patient monitoring and reducing exacerbation-related hospitalisations in a small pilot study [40]. Self-administration is likely to be beneficial for subsets of AATD patients and could help reduce costs associated with treatment. Self-administration with increased intervals between infusions has been trialled in AATD and reduced the annual cost of AAT therapy [41]. Furthermore, self-administration is performed safely in other disease areas, such as haemophilia and immunoglobulin deficiencies $[42,43]$. Overall, the physicians surveyed supported these measures to help improve convenience for patients.

\section{Limitations}

Data were gathered from a survey of AATD experts and do not represent clinical findings. All data (unless otherwise specified) are estimates based on individual physician experience, and do not necessarily reflect actual regional policy and patient demographics. Nevertheless, all physicians selected for the survey were experts in the field of AATD, and were thus well positioned to provide an overview of the status of AATD diagnosis/management in their respective countries based on their clinical experience. Although a good cross-section of Europe is represented, in most cases, only one expert physician from each country returned a response. Further studies including more responses from each country would be required to verify the data.

\section{Conclusions}

This physician survey strongly indicates that AATD remains underdiagnosed and undertreated in Europe. More initiatives are required to support disease awareness and encourage screening of individuals with COPD, adult-onset asthma or bronchiectasis and family members of diagnosed patients. The availability of AAT therapy varies greatly between European countries; cooperation is essential to generate data that could support access to treatment, and to raise awareness of successes in countries where the treatment is available. Improving convenience of AAT therapy is an ongoing objective.

Conflict of interest: I. Horváth reports personal fees from AstraZeneca, GSK, Sandoz, Sager Pharma, Orion and Affidea, personal fees and non-financial support from Boehringer-Ingelheim, Berlin-Chemie, Novartis, CSL Behring, Roche and 
Teva, and non-financial support from MSD, outside the submitted work. J. Chlumský reports fees for lectures and consultancy from CSL Behring. J. Chorostowska-Wynimko reports grants, personal fees, nonfinancial support and other support from Grifols and CSL Behring, personal fees from Kamada, and other support from Shire, outside the submitted work. L. Corda reports personal fees from Grifols and CSL Behring for lectures, speakers' bureaus and expert testimony. J.H. Ficker reports grants from CSL Behring during the conduct of the study; and personal fees from Grifols, and personal fees and non-financial support from CSL Behring, outside the submitted work. M. Kneussl has nothing to disclose. M. Miravitlles reports speaker's fees from AstraZeneca, Boehringer Ingelheim, Chiesi, Cipla, Menarini, Rovi, Bial, Zambon, CSL Behring, Grifols and Novartis, consulting fees from AstraZeneca, Boehringer Ingelheim, Chiesi, GlaxoSmithKline, Bial, Gebro Pharma, CSL Behring, Laboratorios Esteve, Mereo Biopharma, Verona Pharma, TEVA, $\mathrm{pH}$ Pharma, Novartis and Grifols, and grants from GlaxoSmithKline and Grifols, outside the submitted work. G. Thabut reports personal fees and non-financial support from CSL Behring, and grants, personal fees and non-financial support from LFB, outside the submitted work. A.M. Turner reports consultancy fees from CSL Behring, grants from Grifols Biotherapeutics, the Alpha 1 Foundation, the ATS Foundation and the Chest Foundation, grants, conference attendance and fees for clinical trial work as investigator paid to her institution from Arrowhead Inc., and fees for work as study investigator paid to her institution from Boehringer, outside the submitted work. N.G. McElvaney reports grants and personal fees from CSL Behring, grants, and personal fees and non-financial support from Grifols, outside the submitted work. E. Derom has nothing to disclose. E. van 't Wout has nothing to disclose. M. Canotilho has nothing to disclose. M. Sucena has nothing to disclose.

Support statement: The physician survey and development of this manuscript was funded by CSL Behring. Medical writing assistance was provided by Steven Foster (Meridian HealthComms Ltd, Plumley, UK), funded by CSL Behring. Funding information for this article has been deposited with the Crossref Funder Registry.

\section{References}

1 American Thoracic Society/European Respiratory Society statement: standards for the diagnosis and management of individuals with alpha-1 antitrypsin deficiency. Am J Respir Crit Care Med 2003; 168: 818-900.

2 Stoller JK, Sandhaus RA, Turino G, et al. Delay in diagnosis of $\alpha_{1}$-antitrypsin deficiency: a continuing problem. Chest 2005; 128: 1989-1994.

3 Miravitlles M, Herr C, Ferrarotti I, et al. Laboratory testing of individuals with severe $\alpha_{1}$-antitrypsin deficiency in three European centres. Eur Respir J 2010; 35: 960-968.

4 Greulich T, Ottaviani S, Bals R, et al. Alpha1-antitrypsin deficiency - diagnostic testing and disease awareness in Germany and Italy. Respir Med 2013; 107: 1400-1408.

5 Esquinas $C$, Barrecheguren $M$, Sucena $M$, et al. Practice and knowledge about diagnosis and treatment of alpha-1 antitrypsin deficiency in Spain and Portugal. BMC Pulm Med 2016; 16: 64.

6 Greulich T, Averyanov A, Borsa L, et al. European screening for alpha-antitrypsin deficiency in subjects with lung disease. Clin Respir J 2017; 11: 90-97.

7 Piitulainen E, Mostafavi B, Tanash HA. Health status and lung function in the Swedish alpha 1-antitrypsin deficient cohort, identified by neonatal screening, at the age of 37-40 years. Int J Chron Obstruct Pulmon Dis 2017; 12: 495-500.

8 Chorostowska-Wynimko J. Targeted screening programmes in COPD: how to identify individuals with $\alpha_{1}$-antitrypsin deficiency. Eur Respir Rev 2015; 24: 40-45.

9 Chorostowska-Wynimko J. Disease modification in emphysema related to alpha-1 antitrypsin deficiency. COPD 2016; 13: 807-815.

10 Corda L, Bertella E, La Piana GE, et al. Inhaled corticosteroids as additional treatment in alpha-1-antitrypsindeficiency-related COPD. Respiration 2008; 76: 61-68.

11 Chapman KR, Burdon JG, Piitulainen E, et al. Intravenous augmentation treatment and lung density in severe $\alpha_{1}$ antitrypsin deficiency (RAPID): a randomised, double-blind, placebo-controlled trial. Lancet 2015; 386: 360-368.

12 McElvaney NG, Burdon J, Holmes M, et al. Long-term efficacy and safety of $\alpha_{1}$ proteinase inhibitor treatment for emphysema caused by severe $\alpha_{1}$ antitrypsin deficiency: an open-label extension trial (RAPID-OLE). Lancet Respir Med 2017; 5: 51-60

13 Sandhaus RA, Turino G, Brantly ML, et al. The diagnosis and management of alpha- 1 antitrypsin deficiency in the adult. Chronic Obstr Pulm Dis 2016; 3: 668-682.

14 Miravitlles M, Dirksen A, Ferrarotti I, et al. European Respiratory Society statement: diagnosis and treatment of pulmonary disease in $\alpha_{1}$-antitrypsin deficiency. Eur Respir J 2017; 50: 1700610

15 Stockley RA, Miravitlles M, Vogelmeier C, et al. Augmentation therapy for alpha-1 antitrypsin deficiency: towards a personalised approach. Orphanet J Rare Dis 2013; 8: 149 .

16 Soy D, de la Roza C, Lara B, et al. Alpha-1-antitrypsin deficiency: optimal therapeutic regimen based on population pharmacokinetics. Thorax 2006; 61: 1059-1064.

17 Blanco I, de Serres FJ, Fernandez-Bustillo E, et al. Estimated numbers and prevalence of $\mathrm{PI}^{\star} \mathrm{S}$ and $\mathrm{PI}^{\star} \mathrm{Z}$ alleles of $\alpha_{1}$-antitrypsin deficiency in European countries. Eur Respir J 2006; 27: 77-84.

18 Blanco I, Bueno P, Diego I, et al. Alpha-1 antitrypsin $\mathrm{Pi}^{\star} \mathrm{Z}$ gene frequency and $\mathrm{Pi}^{\star} \mathrm{ZZ}$ genotype numbers worldwide: an update. Int J Chron Obstruct Pulmon Dis 2017; 12: 561-569.

19 de Serres FJ. Alpha-1 antitrypsin deficiency is not a rare disease but a disease that is rarely diagnosed. Environ Health Perspect 2003; 111: 1851-1854.

20 Bradi AC, Audisho N, Casey DK, et al. Alpha-1 antitrypsin deficiency in Canada: regional disparities in diagnosis and management. COPD 2015; 12: Suppl. 1, 15-21.

21 Barrecheguren M, Monteagudo M, Simonet P, et al. Diagnosis of alpha-1 antitrypsin deficiency: a populationbased study. Int J Chron Obstruct Pulmon Dis 2016; 11: 999-1004.

22 Bals R, Koczulla R, Kotke V, et al. Identification of individuals with alpha-1-antitrypsin deficiency by a targeted screening program. Respir Med 2007; 101: 1708-1714.

23 Carroll TP, O'Connor CA, Floyd O, et al. The prevalence of alpha-1 antitrypsin deficiency in Ireland. Respir Res 2011; 12: 91. 

in COPD patients. Eur Respir J 2005; 26: 616-622. Molina J, Flor X, García R, et al. The IDDEA project: a strategy for the detection of alpha-1 antitrypsin deficiency in COPD patients in the primary care setting. Ther Adv Respir Dis 2011; 5: 237-243.

26 Corda L, Medicina D, La Piana GE, et al. Population genetic screening for alphal-antitrypsin deficiency in a high-prevalence area. Respiration 2011; 82: 418-425.

27 Chorostowska-Wynimko J, Bakuła A, Kulus M, et al. Standards for diagnosis and care of patients with inherited alpha-1 antitrypsin deficiency Recommendations of the Polish Respiratory Society, Polish Society of Pediatric Pulmonology and Polish Society of Pediatric Gastroenterology. Pneumonol Alergol Pol 2016; 84: $193-202$.

28 Casas F, Blanco I, Martinez MT, et al. Indications for active case searches and intravenous alpha-1 antitrypsin treatment for patients with alpha-1 antitrypsin deficiency chronic pulmonary obstructive disease: an update. Arch Bronconeumol 2015; 51: 185-192.

29 Campos MA, Diaz AA. The role of computed tomography for the evaluation of lung disease in alpha-1 antitrypsin deficiency. Chest 2018; 153: 1240-1248.

30 Green CE, Parr DG, Edgar RG, et al. Lung density associates with survival in alpha 1 antitrypsin deficient patients. Respir Med 2016; 112: 81-87.

31 Edgar RG, Patel M, Bayliss S, et al. Treatment of lung disease in alpha-1 antitrypsin deficiency: a systematic review. Int J Chron Obstruct Pulmon Dis 2017; 12: 1295-1308.

32 Ficker JH, Chapman KR, Turner AM, et al. Alpha 1 antitrypsin $\left(\mathrm{A}_{1}-\mathrm{PI}\right)$ treatment slows emphysema progression independent of baseline FEV1. Eur Respir J 2017; 50: Suppl. 61, OA3416.

33 Rahaghi FF, Miravitlles M. Long-term clinical outcomes following treatment with alpha 1-proteinase inhibitor for COPD associated with alpha-1 antitrypsin deficiency: a look at the evidence. Respir Res 2017; $18: 105$.

34 Campos M, Geraghty P, Holt G, et al. The biological effects of double dose augmentation therapy for subjects with alpha-1 antitrypsin deficiency. Am J Respir Crit Care Med 2017; 195: A6315.

35 Greulich T, Chlumsky J, Wencker M, et al. Safety of bi-weekly intravenous therapy with alpha-1 antitrypsin. Eur Respir J 2017; 50: Suppl. 61, PA710.

36 European Parliament Directorate-General for Internal Policies. EU Options for Improving Access to Medicines www.europarl.europa.eu/RegData/etudes/STUD/2016/587304/IPOL_STU(2016)587304_EN.pdf Date last updated August 2016. Date last accessed: October 242017.

37 European Patients Forum. Directive on Patients' Rights in Cross-Border Healthcare - EPF Position Statement April 2016. www.eu-patient.eu/globalassets/policy/cross-borderhealthcare/epf_position_statement_cbhc_220416.pdf Date last updated: April 2016. Date last accessed: February 18, 2019.

38 Gøtzsche PC, Johansen HK. Intravenous alpha-1 antitrypsin augmentation therapy for treating patients with alpha-1 antitrypsin deficiency and lung disease. Cochrane Database Syst Rev 2016; 9: CD007851.

39 Gauvain C, Mornex JF, Pison C, et al. Health-related quality of life in patients with alpha-1 antitrypsin deficiency: the French experience. COPD 2015; 12: Suppl. 1, 46-51.

40 Wilke A, Semper H, Gross C, et al. Langzeit-Augmentationstherapie von Patienten mit Alpha-1-Antitrypsin-Mangel in der häuslichen Pflege. [Longterm Homecare Augmentation Program in Alpha-1-Antitrypsin Deficient Patients]. Pneumologie 2018; 72: 590-597.

41 Piitulainen E, Bernspång E, Bjorkman S, et al. Tailored pharmacokinetic dosing allows self-administration and reduces the cost of IV augmentation therapy with human $\alpha_{1}$-antitrypsin. Eur J Clin Pharmacol 2003; 59: $151-156$.

42 Schrijvers LH, Beijlevelt-van der Zande M, Peters M, et al. Learning intravenous infusion in haemophilia: experience from the Netherlands. Haemophilia 2012; 18: 516-520.

43 Ochs HD, Gupta S, Kiessling P, et al. Safety and efficacy of self-administered subcutaneous immunoglobulin in patients with primary immunodeficiency diseases. J Clin Immunol 2006; 26: 265-273. 